\title{
El conductismo y algunas implicaciones de lo que significa ser conductista hoy
}

\author{
Camilo Hurtado P.* \\ Universidad Nacional de Colombia
}

Recibido: marzo 24 de 2006

Revisado: abril 28 de 2006

Aceptado: mayo 12 de 2006

\section{Resumen}

Se discuten varios aspectos relacionados con el conductismo, sus múltiples versiones, una definición amplia de éste y a quienes se les puede denominar actualmente "conductistas". Además, a partir de una anécdota se analiza, en términos de barreras epistemológicas, el rechazo a esta tradición de pensamiento y se discute como dicho rechazo está enraizado y mantenido en una serie de malentendidos y en las contradicciones que existen entre los principios del conductismo genérico y los de la llamada "resto de la psicología" o "mainstream psychology" la cual se caracteriza por diversas formas de cognoscitivismo y ser muy afín con la que se ha llamado "psicología popular". Finalmente, se analizan algunas propuestas que han sido sugeridas para superar las barreras epistemológicas del conductismo, concluyendo que el contexto académico actual no es el apropiado para que estos intentos cumplan con su finalidad, en cambio, al parecer dicho contexto es fuente en sí mismo de otro tipo de barreras.

Palabras clave: conductismo, análisis experimental del comportamiento, análisis conceptual del comportamiento, epistemología de la psicología.

* Correspondencia: Camilo Hurtado, Candidato a Magíster en psicología, Universidad Nacional de Colombia. Correo electrónico: camilo_hurtado@msn.com. Dirección postal: A.A. 33842, Bogotá, Colombia 


\section{Abstract}

In this paper are discussed several aspects related to the behaviorism, its multiple versions, an ample definition of this and those who it is possible to be denominated "behaviorists" at the moment. In addition, the rejection to this tradition of thought is analyzed in terms of epistemologic barriers from an anecdote and it is discussed how this rejection is taken root and maintained in a series of misunderstandings and contradictions that exist between the principles of the generic behaviorism and the called "mainstream psychology" which is characterized by diverse forms of cognoscitivism and being very compatible with the denominated "popular psychology". Finally, some proposals that have been suggested to overcome the behaviorism epistemologic barriers are analyzed, concluding that the present academic context is not the appropriate one so these attempts fulfill their purpose, however apparently this context is source in itself of another type of barriers.

Key words: Behaviorism, Experimental Analysis Behavior, Conceptual Analysis Behavior, epistemology of psychology.

En este escrito, intentaré describir las actividades (tales como hacer, decir, escribir, creer, buscar, investigar, teorizar, etcétera) y las razones en función de las cuales se podría decir que alguien pertenece actualmente a una familia o clase llamada "conductista". Se discutirá cómo el actuar "conductistamente" no es "políticamente correcto" en un contexto académico que rechaza esta manera de actuar; intentaré argumentar que dicho rechazo surge al parecer de una serie de malas interpretaciones del conductismo enraizadas en una de las formas más predominantes de "psicología tradicional o popular" (tal como la define Malone, 2001).

Presentaré, primero, una anécdota que ejemplifica el rechazo de la comunidad académica hacia el conductismo: exploré una serie de implicaciones derivadas de dicho rechazo; posteriormente, procuraré aclarar uno de los principales malentendidos respecto a la tradición conductista, para ello daré una definición amplia de "conductismo" aclarando que difícilmente se puede afirmar que existe sólo uno sino más bien múltiples versiones de éste; y finalmente discutiré algunas alternativas que se han venido sugiriendo para reducir el efecto de las barreras epistemológicas al conductismo, concluyendo que aun no disponemos de estrategias completamente poderosas para cumplir con ese objetivo, pues el contexto académico actual no es coherente con ellas y constituye en sí mismo, fuente de barreras adicionales.

\section{Para empezar, una anécdota}

Alguna vez tuve la oportunidad de asistir a un evento de estudiantes de psicología cuyo tema central fue el lenguaje. Como era de esperarse, y debido precisamente al tema del evento, la programación fue realmente heterogénea en cuanto a temáticas y enfoques. El amplio número de charlas dedicadas a temas como la construcción de la realidad a través del lenguaje, herramientas de resignificación, análisis del discurso, entre otros, hizo evidente el peso que tuvieron ciertas aproximaciones al estudio del lenguaje versus tres o cuatro sesiones que hicieron alusión a otras tradiciones. Una de dichas sesiones de la minoría estuvo dedicada a una revisión de una alternativa para el estudio de los fenómenos del lenguaje y la cognición llamada "Teoría 
de los marcos relacionales" (Hayes, Barnes-Holmes $\&$ Roche, 2001). Tal como lo señalaba en aquella ocasión el expositor (un estudiante de psicología al igual que todos los que estábamos allí en aquel momento), podemos ubicar esta aproximación teórica bajo la tradición del análisis del comportamiento, puesto que sus orígenes se remontan a autores bastante representativos como B. F. Skinner. Acercándose el fin de la sesión, el expositor que, hasta el momento se había mostrado muy seguro, tuvo que enfrentar una situación bastante complicada: alguien en el auditorio de manera astuta e inesperada le preguntó ¿es usted conductista?... La aterradora pregunta no sólo llevó a que el estudiante titubeara por primera vez durante toda la charla y se viera obligado a hacer una tortuosa pausa antes de responder, sino que además ocasionó una serie de comentarios, risas y expectativa en el auditorio. Pasados algunos segundos, el consternado expositor en un tono muy conciliador decidió no comprometerse con una respuesta afirmativa; prefirió, en cambio, aclarar que varios elementos de la propuesta teórica de los marcos relacionales le interesaban. Dichas estas palabras, el auditorio pudo respirar de nuevo en paz y allí terminó todo el asunto (Hurtado, 2003).

Varias implicaciones pueden señalarse de este episodio; entre ellas, podríamos preguntarnos ¿qué hubiera ocurrido si el expositor aceptase ser conductista?, ¿por qué tanto recelo a esta tradición?, ¿es posible compartir teorías con énfasis en el estudio del comportamiento sin ser "conductista" o viceversa?, ¿qué ha ocurrido con el conductismo desde aquellos años de la revolución cognoscitiva? Antes de pasar a responder algunas de estas inquietudes considero que resulta fundamental aclarar qué se entiende por conductismo y de allí derivaremos "qué es un conductista". Quizás una parte de la comunidad psicológica mantiene un rechazo hacia algo que realmente no es lo que cree que es o que le "dijeron que era".

\section{Conductismo, conductismos y conductistas}

Siguiendo a autores como Zuriff (1985) y O'Donohue y Kitchener (1999) es posible considerar el conductismo como el nombre apropiado para referirse a un conjunto de tradiciones de pensamiento en psicología y, en algunos casos, en filosofía y ciertos enfoques terapéuticos. A pesar de las malas interpretaciones, el conductismo debe ser entendido como un armazón conceptual en vez de considerarlo como una ciencia o teoría en sí misma.

Existe un acuerdo más o menos generalizado acerca de que son varios los tipos de conductismo que han surgido, se han modificado y conviven actualmente en el trasfondo de las teorías conductuales contemporáneas (Moore, 1998, O’Donohue y Kitchener, 1999 y Zuriff, 1985); si se toma como ejemplo la reconstrucción que hacen O'Donohue y Kitchener (1999) es posible identificar más de 10 formas de conductismo desde los tiempos de Watson hasta nuestros días; la lista contempla el conductismo de Tolman, Hull y Skinner, el interconductismo y la psicología interconductual de Kantor, el conductismo teleológico de Rachlin, empírico de Bijou, teórico de Staddon y biológico de Timberlake, el contextualismo funcional de Hayes, e inclusive de manera sorprendente, se incluyen como formas de conductismo algunos de los aspectos del pensamiento de Wittgenstein, Ryle y Quine. Intentar profundizar en cada una de estas posturas va más allá del objetivo de este texto, sin embargo, el lector interesado podrá encontrar en el manual de O'Donohue y Kitchener (1999) una presentación muy apropiada de cada uno de ellas.

No obstante, a pesar de lo señalado anteriormente acerca de la multiplicidad de "conductismos", varios autores mantienen que en un sentido más amplio, y con relación al resto de la psicología moderna, es posible distinguir primordialmente entre dos formas de conductismo: el radical skineriano y el metodológico. En este sentido, autores como Malone (2001) defienden que la psicología a partir del siglo XX tomó al menos dos versiones: una minoritaria y tendiente a ser afín, o derivada del conductismo radical fundando por B. F. Skinner (por ende, varias de las formas de conductismo que presentan O'Donohue y Kitchener estarían incluidas en esta minoría), y una mayoritaria (llamada por Malone como el "resto de la psicología"), que también, a pesar de sus múltiples versiones, podría 
decirse que tiene como característica principal ser mediacional ("teórica" como la llamó B. F. Skinner) o inclusive como el mismo Malone (2001) sugiere, plantear que se trata de múltiples subdivisiones de una "psicología cognoscitiva" que acuden a explicaciones expresadas en términos de mecanismos subyacentes y que, en algunos casos, aún mantiene formas de dualismo ontológico mente-cuerpo.

Bajo la anterior distinción aparece uno de los malentendidos quizás más enraizados en la psicología, el cual consiste primordialmente en asociar el conductismo metodológico con la postura skineriana. Como señala Malone (2001), tal asociación es completamente equivocada pues el mismo B. F. Skinner en varios de sus textos precisamente criticó esta forma de entender los fenómenos psicológicos, especialmente aquellos relacionados con lo que ha sido denominado genéricamente "cognición" (e. g. los eventos privados y el pensamiento). Podría irse más allá y afirmarse que las formas de conductismo metodológico realmente tienden a ser más coherentes con algunas de las posturas incluidas en la forma mayoritaria del "resto de la psicología". Tómese como ejemplo el operacionalismo utilizado en algunas orientaciones psicológicas para definir los fenómenos psicológicos "inobservables" a partir de "conductas observables", tal tipo de concepción sería completamente coherente con una postura metodológicaconductista (Moore, 1998) y completamente incompatible con una aproximación radical skineriana y, por ende, con varias de las formas afines derivadas incluidas en el listado del manual de O'Donohue y Kitchener (1999).

Autores como Zuriff (1985) sugieren que es posible subrayar los componentes que permiten delimitar la estructura conceptual de una versión genérica del conductismo, estructura que tendería a excluir las formas de conductismo metodológico y, en cambio, ser muy afín con la forma radical y sus posteriores desarrollos. Para Zuriff (1985), dicha versión amplia o genérica del conductismo es una red integrada de supuestos, que como un "aire de familia” (según el concepto de Wittgenstein), están presentes en la gran mayoría de los conductismos. Los cuatro componentes que nos sugiere Zuriff (1985) son:

1. El conductismo es la filosofía de la ciencia del comportamiento (análisis del comportamiento). Antes que cualquier hallazgo empírico, la postura conductista dicta los cánones acerca de qué tipo de preguntas psicológicas son válidas y que métodos son aceptables en la búsqueda de sus respuestas. Hace, además, explícitos los criterios para la adecuación de una explicación científica.

2. El conductismo es una filosofía de la mente, con ciertos supuestos acerca de la naturaleza humana. Esta filosofía de la mente es interdependiente con la filosofía conductista de la ciencia. Una ciencia restringida a un grupo limitado de métodos y formas de explicar, tenderá a reafirmar una concepción particular de lo mental (usualmente antidualista).

3. Es además importante, como componente de la estructura del conductismo genérico, que se intente reconstruir el reconocimiento de una serie de supuestos empíricos acerca de la conducta de los organismos, su relación con el ambiente y la efectividad de varios métodos de investigación científica y construcción teórica por encima de otros.

4. Por último, el conductismo representa un conjunto de valores. Recomienda las metas para una ciencia del comportamiento y sugiere algunos estándares para evaluar la actividad científica. La importancia de esta "ideología" es mayor en las áreas de estudio del comportamiento con énfasis aplicado, en las cuales el conductismo promueve intervenciones congruentes con intereses sociales.

A partir de los aspectos que señala Zuriff es evidente que cada forma de conductismo podrá tener concepciones distintas o similares frente a cada uno de los cuatro aspectos anteriormente señalados, por lo tanto una compleja red de aspectos compartidos podría dar pie a reconstruir dicho "aire de familia". 
Quedaría clara hasta acá una posible delimitación del conductismo o de los "conductismos", pero sigue quedando pendiente el asunto de los "conductistas". Ser conductista no es "tener algo", de la misma forma que hablar de conductismo no implica decir que existe un objeto o evento independiente de las prácticas de una comunidad académica que "causa" el decir cosas "imprudentes" en contextos académicos.

Propiamente ser conductista significaría desplegar cierto tipo de actividades, en otras palabras, comportarse de ciertas maneras (hacer, decir, escribir, creer, buscar, investigar, teorizar, etc.). Por otra parte, decir que alguien es conductista es comportarse frente a la conducta de otro y ajustarse a la convención de una comunidad que establece responder de cierta manera ante el comportamiento de otro individuo. Para ambos casos, el del "actor" conductista y el del observador que nomina y rechaza la conducta del conductista, alguien coherente con la tradición filosófica que se reseñó anteriormente se preguntaría que contingencias han mantenido ambos comportamientos.

Es así como una importante pregunta de investigación hipotética estaría enfocada a indagar cómo el actuar "conductistamente" se sigue manteniendo a pesar del constante rechazo de la comunidad, mientras que otra pregunta se relacionaría con la exploración del porqué la nominación y el rechazo conductista que presentan los miembros de la comunidad psicológica tradicional no han cambiado a pesar de los múltiples esfuerzos realizados por quienes han representado la tradición conductual más adelante en el presente documento se plantean esbozos de respuesta para ambas preguntas hipotéticas.

Las maneras de comportarse son diversas, pero se supone que a pesar de esas variaciones el aire de familia conductista debería poderse seguir evidenciando de forma más o menos acentuada. Sin embargo, aparecen una serie de inconsistencias, que bastante estudiadas por los analistas del comportamiento, se ejemplifican en la anécdota del expositor señalada al comienzo del texto: algunas personas se comportan como conductistas pero no dicen serlo, otras, en cambio, dicen ser conductistas pero sus maneras de actuar son opuestas, en otras palabras, hay una inconsistencia entre el "hacer" y el "decir". Un analista del comportamiento probablemente diría que dicha contradicción deriva de que el "decir" está mantenido por ciertas relaciones que establecen los individuos con su contexto (contingencias) mientras que el "hacer" por otras; podemos entonces preguntarnos ¿cuáles podrían ser dichas formas de interactuar con el entorno que favorecen dichas contradicciones?

Como lo señalan O'Donohue y Kitchener (1999), actuar como conductista genera fuertes reacciones negativas en quienes presencian dichos actos, las razones parecen ser varias, pero una de ellas es la contradicción entre los principios conductistas y algunas versiones de la "psicología popular" o "versión tradicional", propuesta por Malone (2001), que entre otros elementos, está formada a partir de la "doctrina oficial", "el dogma del fantasma en la maquina" o "la teoría de la doble vida" según las cuales con la dudosa excepción de los niños de brazos y los idiotas, todo ser humano tiene en conjunto, un cuerpo y una mente (Ryle, 1967). Por lo tanto, hacer una psicología humana, con un énfasis en la experimentación, la predicción y el control, asumiendo además una postura crítica acerca del mentalismo y el dualismo, es perfectamente lo menos "apropiado" a la vista de una comunidad psicológica con metas analíticas diferentes y que, según las múltiples deformaciones y malas interpretaciones, ha asumido, entre otras cosas, que el conductismo murió hace más de 40 años y que es anti-humanista. En nuestra anécdota, el expositor actúo conforme las contingencias lo han establecido, anticipando el castigo por actuar según la tradición.

Qué paradójico, el analista del comportamiento puede explicar por qué él no puede hablar frente a sus colegas y puede explicar por qué ellos reaccionarán de cierta manera, pero no puede decirlo porque se cumple la predicción. 


\section{El porqué del rechazo y las alternativas}

Hasta acá ha sido descrita la versión genérica del conductismo siguiendo a autores como Zuriff (1985) y O’Donohue y Kitchener (1999), además, en función de dicha definición se ha planteado a quién sería apropiado catalogar como miembro de la amplia familia "conductista". Sin embargo, sólo se ha descrito brevemente una de las razones por las cuales el "conductismo" ha continuado siendo visto negativamente por el resto de la comunidad psicológica que ha seguido el "resto de la psicología" o el "mainstream" (Malone, 2001). En el anterior apartado se mencionó el problema de la "doctrina oficial" de la doble vida del cuerpo y la mente, y la psicología popular que está asociada a ella. A continuación se discute con mayor detalle este último punto.

O’Donohue y Kitchener (1999) señalan algunas características de la visión no conductista del conductismo. Entre ellas señalan los errores de interpretación (exégesis) de la literatura conductista, las reacciones emocionales negativas, la asociación equivocada entre algunas tradiciones filosóficas superadas (p. e. el positivismo lógico) y el conductismo, entre otras. Sin embargo, una que llama bastante la atención es aquella relacionada con la contradicción entre la psicología popular y algunos elementos de la visión conductista (O’Donohue, Callaghan y Ruckstuhl, 1998). Al parecer las caricaturas irónicas del conductismo, y en general, todo el rechazo a cualquier forma de conductismo es el resultado de algo que es aún más fundamental: la psicología popular y su elemento definitorio, la doctrina dualista de la doble vida mente-cuerpo.

La preocupación fundamental que enfrentan O'Donohue y cols. (1998) en su texto es dar cuenta del porqué los estudiantes de psicología, al fin y al cabo los futuros miembros de la comunidad académica y los que con su práctica permiten o no la existencia de determinada tradición de pensamiento independientemente de su validez o grado de verdad, tienen dificultades con aproximarse al conductismo radical en comparación con la psico- logía cognoscitiva ("el resto de la psicología" según Malone, 2001). La explicación según estos autores radica en el conocimiento que traen los estudiantes a la universidad, conocimiento que han ganado en su vida no académica desde temprana edad en su intercambio con la normatividad social "no científica" y que dada su "utilidad" en la vida diaria para entender la realidad, pocas veces es cuestionado, se toma como dado y para muchos es "obvio". Este tipo de conocimiento es a lo que varios autores, siguiendo el concepto de G. Bachelard (1884-1962) han llamado una barrera epistemológica y, en última instancia, supone que los investigadores de cualquier rama científica siempre van a verse limitados más o menos por este tipo de saberes.

Antes que cualquier formación en psicología llegue a darse, usualmente las personas (tanto estudiantes, profesionales de psicología o las personas ajenas a la disciplina) parecen funcionar bajo una teoría general psicológica (psicología popular) que puede resumirse en los siguientes aspectos: (a) las creencias, intenciones y deseos causan lo que la gente hace, por lo tanto, constituyen los elementos predictores y explicatorios por excelencia; (b) dichas intenciones, deseos, creencias, etcétera, constituyen causas internas, no observables directamente por otras personas, por lo tanto, el papel de las personas entrenadas, entre ellas los psicólogos o los "sabios", precisamente, consiste en develarlas, como quien puede buscar o construir la llave correcta que gira en la cerradura y permite acceder a un mundo oculto detrás de las apariencias de la conducta observable (Ryle, 1967); (c) dada la complejidad de lo humano, difícilmente una única teoría, con un conjunto pequeño de elementos explicativos, puede dar cuenta de tan complicado asunto; (d) siempre existe la posibilidad que la "voluntad" de las personas cause lo que hacen, el concepto de libre albedrío se enfrenta usualmente a cualquier intento de establecer leyes acerca de lo que la gente hace y usualmente sirve en esta teoría psicológica popular para explicar aquellos casos donde las personas no actúan conforme se espera; finalmente se menciona que (d) la teoría popular contempla implícitamente, y en algunos casos explícitamente, que los "verdaderos" méto- 
dos científicos hacen uso de diseños experimentales de grupo (O’Donohue y cols, 1998).

A partir de estos elementos de la psicología popular, O'Donohue y cols. (1998) argumentan que el camino hacia la vinculación al juego de la psicología científica cognoscitiva (o la psicología tradicional) es de "mínima resistencia", mientras que el camino hacia el juego del conductismo (primordialmente en su forma radical o las versiones afines) perfectamente puede ser descrito como "tortuoso" puesto que quizás el único aspecto que puede llegar a ser contradictorio a la psicología popular, desde el punto de vista cognoscitivo, es el asunto de la voluntad y el determinismo ${ }^{1}$, pero de ninguna manera los obstáculos son tantos como en el caso del conductismo radical, el cual definitivamente contempla contradicciones con respecto a todos los elementos.

Pero bien, ¿qué hacer?, se preguntan estos autores. La solución a este problema puede resumirse en estos cuatro puntos: (a) debe hacerse explícito el conflicto entre el saber previo (barrera epistemológica) y el que está representado por la psicología conductual, (b) además, se sugiere que puede ser útil mostrar cómo en la historia de la ciencia son muy frecuentes los casos en los cuales los hallazgos científicos son contraintuitivos, y que la psicología no es la excepción, (c) por otra parte, se propone ofrecerle a las personas que se acercan al análisis del comportamiento las diferentes perspectivas propias de las psicologías conductuales y cognoscitivas y, finalmente, (d) se propone que demostrar el poder del análisis conductual para aplicarse empírica y conceptualmente a diferentes áreas, entre ellas la clínica puede ser apropiado.
Este último asunto de las alternativas a primera vista parece "atractivo", sin embargo, en un contexto académico como en el que se enmarca esta discusión, difícilmente podría vérsele como viable, porque nuevamente supone aspectos que no hacen parte del "juego"que actualmente está presente en la "comunidad académica psicológica".

A continuación se presentan algunos aspectos que, a juicio del autor, dejan en entredicho las alternativas de O'Donohue y cols.(1998): (a) la noción de ciencia en psicología pareciera estar actualmente desplazada hacia una perspectiva que difícilmente aceptaría que los criterios de predicción y control sean los más válidos, así las cosas, mostrar la utilidad de los asuntos conductistas no necesariamente sería apropiado, probablemente podría ser visto como propio de una versión de ciencia retrógrada y nuevamente vendría el ya bastante común rechazo; (b) en relación con lo anterior, otro problema es que el criterio de verdad vigente en las instituciones encargadas de regular la práctica científica y de asignar recursos para que los científicos coman y duerman tranquilos, y así tengan tiempo para formular preguntas interesantes; han asumido un criterio de verdad más asociado con la relevancia social de cualquier producto científico, razón por la cual difícilmente cualquier intento de seducción hacia el lado de la práctica en análisis experimental del comportamiento vendrá a ser reforzado, pues la normatividad lo castiga no adjudicando recursos y criticándolo; c) otro problema, quizás el más grave, tiene que ver con los intereses de la formación en psicología.

Para nadie es un secreto que la oferta cada vez es más profesionalmente orientadas y esto no es algo

\footnotetext{
En este sentido O'Donohue y cols. (1998) argumentan que tanto la psicología conductista de tipo radical y sus versiones afines o derivadas, como también algunas de las psicologías cognoscitivas, usualmente se ven enfrentada a los mismos obstáculos o "barreras" epistemológicas propios de la forma de psicología popular más difundida: dichos obstáculos son el problema de la voluntad o libre albedrío y el determinismo.

Tanto conductistas como cognoscitivistas interesados en formar nuevos psicólogos o divulgar el conocimiento psicológico propio de sus enfoques, usualmente deben superar concepciones muy arraigadas acerca de la posibilidad de que el comportamiento humano se encuentre sometido a algún tipo de ley o regularidad que permita predecir que va a decir o a hacer una persona dadas determinadas circunstancias; de igual forma también conductistas y cognoscitivistas deben confrontar el problema de la "voluntad" como entidad que es causa última y fundamental de las acciones de las personas; en este sentido el viejo chiste popular que pregunta "cuantos psicólogos se necesitan para cambiar un bombillo" y al cual se responde de forma supuestamente graciosa diciendo que "no importa el número, si el bombillo no quiere cambiar"... expresa como la psicología popular supone que siempre existe la posibilidad de explicar porque las personas actúan acudiendo a su "fuerza de voluntad", “libre albedrío", etcétera.
} 
azaroso, pues al fin y al cabo la misma "teoría popular" asume que la psicología es profesión más que ciencia, así las cosas, iqué más obstáculo puede haber que querer volver científico al que no quiere!

\section{Referencias}

Hayes S. C., Barnes-Holmes, D. \& Roche, B. (2001). Relational frame theory: a post-skinnerian account of human language and cognition. New York: Kluwer Academic/Plenum Publishers.

Hurtado, H. C. (2003). ¿Conductista yo? De-mente. 1, 23-26.

Malone, J. C. (2001) Radical behaviorism and the rest of psychology: a review/précis of skinner's about behaviourism. Behavior and Philosophy, 29, 31-57

Moore, J. (1998). Behaviorism Tutorial. Recuperado el 23 de mayo de 2005, de la Universidad de Athabasca (Cánada): http:// psych.athabascau.ca/html/Behaviorism

O'Donohue, W. T. \& Kitchener, R. (1999) Handbook of behaviorism. New York: Accademic Press.

O’Donohue, W. T., Callaghan. G. M., and Ruckstuhl, L. E. (1998). Epistemological barriers to radical behaviorism. The Behavior Analyst, 21,

Ryle, G. (1967) El concepto de lo mental. Buenos Aires: Paidós.

Zuriff, G. E. (1985). Behaviorism: A conceptual reconstruction. New York: Columbia. 